\title{
Understanding the hermeneutics of digital materiality in contemporary architectural modelling: a material engagement perspective
}

\author{
Kåre Stokholm Poulsgaard ${ }^{1}$ - Lambros Malafouris ${ }^{2}$
}

Received: 15 December 2019/ Accepted: 1 August 2020

(c) The Author(s) 2020

\begin{abstract}
This article develops a framework for analysing how digital software and models become mediums for creative imagination in architectural design. To understand the hermeneutics of these relationships, we develop key concepts from Material Engagement Theory (MET) and Postphenomenology (PP). To push these frameworks into the realm of digital design, we develop the concept of Digital Materiality. Digital Materiality describes the way successive layers of mathematics, code, and software come to mediate enactive perception, and the possibilities of creative material engagement actualised in work with software. Just as molecular materials come to transform action with material objects, so digital materiality comes to enable and transform creative practices with computers. Digital architectural design form a new space for ongoing enactive discovery and creativity through manipulation of digital models and their underlying software environments. By shifting relationships within their digital models, architects can direct their attention, intention, and imagination towards widely different aspects of the model. Here, creative imagination becomes a fundamentally situated activity where mind emerges through dynamic interaction between a variety of embodied, material, and cultural domains.
\end{abstract}

Keywords Postphenomenology $\cdot$ Material engagement theory $\cdot$ Architecture $\cdot$ Design $\cdot$ ICT $\cdot$ Parametric $\cdot$ Material culture

\section{Digital design in a postphenomenological perspective}

In contemporary architecture, computational tools let designers build intricate digital models where geometry translates to data, and data to geometry. These models allow for intuitive creation of advanced 3D geometries, fluid shapes, and complex construction details. They integrate the mathematics and numerical calculations that underlie these into computational environments that expand from being

Lambros Malafouris

lambros.malafouris@arch.ox.ac.uk

Kåre Stokholm Poulsgaard

kare.poulsgaard@sjc.ox.ac.uk

1 St John's College and Institute for Science Innovation and Society, University of Oxford, 64 Banbury Road, OX2 6PN Oxford, United Kingdom

2 Hertford College and Institute of Archaeology, University of Oxford, 36 Beaumont Street, OX1 2PG, Oxford, United Kingdom design tools to also becoming instruments for creative discovery for architects.

What, then, is the role of software in architectural design? Who designs, the architect or the digital tool? How can we begin to understand the computational environment as a medium for enactive discovery (Malafouris 2011) and creative material engagement (Malafouris 2014, 2019, 2020; Koukouti and Malafouris 2020; Poulsgaard and Malafouris 2017; Poulsgaard 2017; Clowes 2018, 2019)? With the growing prevalence of software in architecture, digitally minded architects are enquiring into the relationship between computational tools and their creative agency (Kolarevic 2003; Terzidis 2006; Picon 2010; Burry 2011; Menges and Ahlquist 2011; Carpo 2011; Oxman and Oxman 2014; Davis 2013b; Rowe 2017). A basic assumption for these discussions is that designers are facing a new technologically mediated terrain in design thinking and that there is a need to formulate a rationale for digital design theory and practice (Oxman 2008:102). What might we learn from these practices and debates in contemporary architecture about the relationship between technology and creative imagination? 
The word technology fuses the Greek root tekhne, which means art or craft, with logos which means discourse or branch of learning. 'Technology is to technics what linguistics is to language, what every science is or would be to its objects,' writes anthropologist François Sigaut (1994:424). In this sense, the word technology denotes the study of technical practices and products, yet this is clearly not our everyday use of the word. As warned by philosopher Don Ihde (1993), we tend to use the word to describe machines, gadgets, and things, but taken in isolation from their social environments of production and use these objects can only be abstractions.

Both Sigaut (1994) and Ihde suggest that we should rather see technology in relation to specific embodied practices reproducing organisational, cultural, and social mores. Technologies mediate our relations with the world around us (Ihde 2009). They must be understood not as isolated objects or machines but as unfolding human-technology relationships in constant and dynamic co-evolution. Ihde uses the word 'technoculture' to describe these relationships, arguing that technology embodies essential aspects of the culture of their production and use (Ihde 1993:57; Tripathi 2017). Technology, in short, is fundamental to what it means to be a human amongst others in the world.

Ihde's (1990, 1993, 2009, 2012) Postphenomenology (PP) argues for analysis of technical experience through the active relational pair of human-technology in all its dimensions. In this lies an anti-Cartesian attack on early modern epistemology and metaphysics, specifically dualisms such as mind/matter, tool/individual, action/context. Instead, PP argues for analysis of how knowledge exists in and as technically mediated embodied practice. Its core assumption is an inter-relational ontology (Ihde 2012) where whatever changes show themselves in the environment qua technology, relate to or call forth responses for the experiencers of that environment. The notion of material hermeneutics (Tripathi 2017; Tripathi forthcoming) describes these relationships and the importance of technical artefacts for our interpretation of objective reality (see also Ihde 1993, 1999, 2009).

When applied to the question of digital modelling and creative imagination in architecture, this perspective highlights how contemporary design practices are essentially embodied in their digital tools. What PP highlights is how design technology — as both tekhne and logos-come to serve as extensions of perceptual and bodily intentionality into synthetic worlds revealed by the increasing intricacy of design software and digital models. These worlds fluidly integrate a multiplicity of scales from the level of masterplans and full building designs through to minute construction details for say a façade element. Ihde urges us to approach these technical relationships with 'a kind of "instrumental realism" regarding the world-referentiality of this new mode of human perceptual embodiment' (Ihde 1993:3). What this means is that the correlate, humantechnology is fundamental to our being in the world, to knowledge, creativity, and agency. In the perspective of $\mathrm{PP}$, technology in a very real sense forms the horizon of our imagination. There is nothing deterministic in the inevitability of this relationship though. As Ihde takes care to highlight, the sheer complexity of these relationships introduces an inherent plurality of perception. As our engagement with tools (tekhne) is embedded within larger organisational, social, and cultural mores (logos), plurality and variability are not accidental features of these relationships but essential. They belong to the very structure of perception qua technology, which can, therefore, only proceed through enactive discovery of both tools and the worlds they give access to (see Malafouris 2011, 2014). Perception, Ihde (1993:87) writes, must always 'learn its ways.'

For contemporary architectural design, this means that we can understand digital models as technologies of perception that are inextricably bound up with knowledge and imagination in the wider field of architecture. Like any technology, these models are embedded within organisational, social, and cultural mores and therefore lend themselves to variability and ongoing enactive discovery. This proceeds through manipulation of the models, through digital material engagement.

\section{Material engagement theory and creative agency}

Here, we draw on the work of Material Engagement Theory (MET) (Malafouris 2013a, 2014, 2015, 2019; Poulsgaard and Malafouris 2017; Poulsgaard 2019, 2020; Walls and Malafouris 2016) to argue that digital design tools have their own materiality, which comes to facilitate architects' creative work through their intrinsic predisposition to variation.

We define the concept of digital materiality (Poulsgaard 2020) to describe the way successive layers of mathematics, code, and software come to mediate and shape creative agency in digital modelling. Just as molecular materials act as resistance and come to transform action upon material objects, so digital materiality comes to enable and transform creative practices upon computers. This has consequences for our concept of creative agency. Malafouris 
(2013a, b) has advanced the notion of material agency to describe how materials and technics actively mediate our possibilities for acting in the world. In this view, agency is the relational and emergent product of material engagement-our work with and through materials, objects, and technology. In line with PP, this relational perspective sees agency (including the ability to imagine and enact new worlds ${ }^{1}$ ) inside human interactions with the material properties of the world. The concept of material agency goes further than material hermeneutics in its implications, however. Where material hermeneutics describes the role played by technologies in mediating our perception of the world, material agency posits that world and human cognition are ontologically inseparable. Where the first seeks to analyse how technologies disclose the world for humans to act upon (see Ihde 1999), the latter posits that agency is not simply dependent on objects and technics but rather an emergent property arising from the dynamic coupling between people and technics. Rather than positing agency as something innate to humans, this perspective seeks to fully restore the power of objects and technics to influence our being and acting in the world. In this perspective, the inextricability of human experience from technology can further explain how architect's creative imagination is tied up with ongoing material engagement (Malafouris 2009, 2011, 2013b, 2014, 2016, 2019).

\subsection{Material engagement theory}

MET provides a reframing of creative agency by situating it within a relational framework of mind and material engagement where mind emerges through dynamic interaction between a variety of embodied, material, and cultural domains. This situated perspective does away with Cartesian dualism by refusing to locate mind either in the head or in the world. Rather it seeks to overcome these and other dualisms (mind/matter, tool/individual, action/context) by showing how elements usually posited on either side of these pairs are entangled in ongoing processes of mind in action.

MET positions itself against representational theories of mind. These theories argue that cognition proceeds through manipulation of mental representations of reality. This reproduces dualisms between internal representations and the external world, mind, and matter.

In representational theories of design, creativity becomes synonymous with mental manipulation of representations

\footnotetext{
${ }^{1}$ See also Stiegler's (1998) discussion of technically mediated anticipation. For Stiegler, we are always extending our bodily and cognitive abilities via technical prostheses. In this perspective, technology is a constantly evolving prosthesis for our creative facilities and therefore inextricably tied to anticipation and imagination.
}

of a given problem with little interference from the outside world - whether material, technical, or social (e.g. Newell et al. 1959; Simon and Newell 1971). This theory of creativity is too limiting for a comprehensive analysis of digital design practices as it leaves little scope for understanding the interplay between architects and digital tools in the specific institutional contexts of the architecture studio.

Against representationalism, MET champions a relational approach to thinking and doing. Creativity and mind emerge through an interplay of forces extending beyond individuals into environment, culture, tools, and materials. This perspective radicalises theories of extended mind (Clark and Chalmers 1998) by collapsing distinctions between internal and external elements of cognition. This follows recent work in the field of enactivist philosophy of mind that propose to move beyond theories of extended mind by rejecting representations while recasting the bounds of cognition; deprived of representations mind becomes enactive, essentially world involving, inherently extensive rather than occasionally extended (Varela et al. 1991; Thompson 2007; Di Paolo 2009; Hutto and Myin 2013; Chemero 2009; Gallagher 2017; Gallagher and Allen 2018). MET shares the anti-representationalism of enactivism and posits that material engagement acts as a fundamental cognitive resource in its own right- that we think with and through things in action. What matters are neither representations nor their mental manipulation but rather how material engagement helps recast difficult problems into more manageable terms that support imaginative solutions (cf. also Brooks 1991; Dreyfus 2002; van Gelder 1995).

MET proposes to move beyond mental representations by developing a concept of the material sign which opens for analysis of enactive signification (Malafouris 2013a). Material signs do not primarily embody a representational logic but an enactive one. They bring forth and instantiate meaning through their very materiality.

Material signs are not mere vehicles for the transmission and communication of meaning, rather they constitute, in their material presence, a hylonoetic field of meaningful action and interaction (from the Greek words (hyle) for matter and (nous) for mind, see Malafouris 2016). In that sense, material signs provide the actual physical forces that shape our social and cognitive universe (see also Malafouris 2019). This depends on a strong commitment to the relational ontology of situated and embodied action. Enactive signification highlights how creative mind rides on movement, affect, and transaction with shifting materials, and how this transaction profoundly shapes the perception and imagination of cognitive agents as well as their ability to design tools, models, and ideas (Koukouti and Malafouris 2020; Malafouris 2019, 2020; see also Rietveld and Brouwers 2017; van Dijk and Rietveld 2018; Pallasmaa 2017). 
MET and PP shares this commitment to a relational ontology in which people and tools are inextricably linked. Both frameworks emphasise practice and experience rather than representations in their analysis and seek to collapse distinctions between making and knowing (Ihde and Malafouris 2019). The two approaches are complementary in the analysis of digital design and mind. MET's focus on cognition and agency works alongside PP's emphasis on case studies of technology to enrich the study of how perception, imagination, and mind are tied up with technics.

\subsection{Material agency}

In line with MET and PP, we see mind as essentially coextensive with technics and matter (see also Poulsgaard and Malafouris 2017; Poulsgaard 2019, 2020). Mind is an emergent and relational process. Neither bound by the head nor the body, mind emerges from interactional ecologies reaching well beyond the individual. What matters is the coextensive entwining of cognitive processes, technics, and material culture in situated action.

This commitment to interactivity has consequences for our concept of creative agency in design. The key epistemological and practical problem becomes accounting for the ways in which different technical and material registers come to co-constitute and extend the flexibility of our minds. In our enactive perspective action does not primarily arise from mental plans and representations, but rather from within the interplay of forces in a given cognitive ecology (see also Hutchins 2010). Agency and imagination become emergent properties that cannot be reduced to either human or non-human components. They become the property of an interactive, relational domain where all elements - technical, social, cultural-have had their power to influence each other restored. The notion of material agency seeks to capture this domain of possibilities and the fluid properties of mind in action across these. It collapses hard distinctions between internal and external cognitive processes and forwards a relational perspective on agency. Malafouris sums up this pragmatic perspective:

'If there is such a thing as human agency, then there is material agency; there is no way human and material agency can be disentangled. Or else, while agency and intentionality may not be properties of things, they are not properties of humans either; they are properties of material engagement.' (Malafouris 2013a:119).

This follows anthropologist Alfred Gell's (1998:20) argument in Art and Agency for the powers of objects to move us, cause, elation, fear, involvement. To advance this argument, Gell attributes agency to the detection of its effects in the causal milieu rather than internal human intentionality. We extend and critique Gell's argument for object agency to argue that agency cannot be the property of a human agent, nor of an object, but can only emerge in their interaction. In this view, agency is the relational and emergent product of material engagement. It is not something given to humans but something to be realised as humans interact with the materials of the world, often mediated through technologies that themselves have material properties. The important question is not "what is agency?' (as a universal property), but rather 'when and how is agency constituted and manifest in the world?' (Malafouris 2013a:147).

Malafouris has put forward the analogy of a potter at the wheel to explain how creativity emerges from the interactions between matter, body, and brain. The potter cannot simply create a pot from an initial mental image of it; the wet clay will resist and transform the potter's actions on it. Instead, the potter must feel and follow the clay as the pot emerges through this dynamic transaction. It is not just the pot that is emerging and undergoing continuous change at the potter's wheel but also the potter's idea of the pot, his creative and cognitive abilities (ibid: 145, see also 2008, 2009, 2014, 2020). MET alerts us to the fact that the creation of form is not a question of abstract ideas being imposed on inert matter; rather it is one of the forms and ideas emerging through continuous interaction, variation, and discovery. We argue that this relational process translates just as well to the digital realm of design models as the molecular world of clay pots. To make this argument we rely on the concept of digital materiality (Poulsgaard 2020).

\section{Digital models and digital materiality in architecture}

Before 1946, 'computer' was a job description. Computation was carried out by people, almost exclusively women, equipped with computation sheets and organised in rows that formalised the step by step execution of algorithms. In the 1940s, this work brought pioneers in computation and computer graphics such as John von Neumann and Isaac Jacob Schoenberg together under the command of the US Army during the early years of the Second World War. The war required bombs and firing tables and developing these weapons required computation on a grand scale. During the war, von Neuman and others realised that digital computers could drastically reduce the time and cost of this work. The first electronic general-purpose computer, the ENIAC, was developed by these people to calculate firing tables and its first program was a feasibility study for the nuclear bomb (Dyson 2012). 


\subsection{Computational tools and architectural design}

The digitisation of computation facilitated an explosion in calculation capacity, which subsequently enabled new approaches to architecture and design (see also Picon 2010; Carpo 2011; Penny 2017). In the early 1960s, a young software engineer at MIT named Ivan Sutherland (1963) wanted to revolutionise human-computer interaction by creating the first interactive Computer-Aided Design program. The program, which he developed as his doctoral thesis, was called Sketchpad. Sketchpad is one of the earliest predecessors to the graphical user interfaces which are ubiquitous today and Sutherland received the Turing Award in 1988 for its development.

Back in 1963, Sutherland made it clear that drawings made with Sketchpad were: 'entirely different from the trail of carbon left on a piece of paper,' since not only the information that gave the drawing its particular appearance (the trace of its lines) was stored in the computer, but also information about how the drawing was made and tied together (the step by step process and numerical calculations underlying the lines) which would allow it to keep its internal consistency during transformation (Sutherland 2003[1963]:25). In this way, Sketchpad introduced a new computational element to architetcural drawing. This no longer consisted of aggregated lines on paper or screen but now also contained numerical descriptions of lines and basic shapes as well as relationships between them. Architect Daniel Davis (2013a, 2013b), explains why this shift from drawing to relationships within the drawing marked a revolution in digital design:

'Sketchpad offered a new way to explore parametric equations $(. .$.$) designers could explore variations by modifying$ parameters and having Sketchpad automatically recalculate and redraw the geometry. But in Sketchpad designers were also free to modify the relationships of the model, which would also cause the recalculation and redrawing of geometry. Thus, the architect's control of Sketchpad, as with most parametric modelling software, is not only through the parameters of the model but also through the model's underlying relationships.' (Davis 2013a).

The keyword here is parametric. Parametric design works by establishing points in 3D digital space before declaring mathematical relationships between these to generate lines or geometry. Once the parametric model has been devised it can be used for endless variations through the manipulation of the linked parameters as well as their mathematical relationships.

A parametric model requires the explicit definition of relationships between parameters within the model. Once these are stated, changes to the parameters or their underlying relationships will change the geometry of the model. In contemporary architectural design, parametric models become environments for the continuous computation of form rather than a notational description of a single form. This capacity utilises the power of mathematical splines, a graphical innovation first introduced by Isaac Jacob Schoenberg following his US Army days, as their underlying polynomial functions deal more easily with the continuous calculation of lines and curves (Carpo 2011:40).

In architecture, this curvilinear continuity was popularised in the 1990s through the work of philosopher Gilles Deleuze (1988), whose discussions of morphology, folds, and topology caught the attention of a new group of digital designers. At the core of Deleuze's work on the fold is a logic of continuous variation resting on polynomial functions. Its presentation as a program for continental philosophy did much to popularise parametric functions within architecture; as architectural historian Mario Carpo (2011:40) dryly notes: 'Without Deleuze's timely mediation, few architects would have found high school calculus so highly inspiring.' Architect Greg Lynn's 1993 essay on "Architectural Curvilinearity" is widely recognised as a foundational moment in a new topological approach to design through parametric computation, and the 1990s saw an explosion of curves, folds, topologies, and blobs in architecture (Carpo 2012; Picon 2010).

The rise of curvilinearity in architecture is a result of what we call digital materiality. It was powered by mathematical splines, the explosion in computation power, and the development of interactive graphical user interfaces for manipulation of form alongside cultural responses to these innovations (see Poulsgaard 2020; Picon 2010).

The logic inherent in parametric design use computers to break away from the additive logic of drawing and drafting, which had been in place since the Renaissance. Instead, parametric equations rely on the explicit description of relationships between elements, thereby introducing an associative logic into the creation of form (Tedeschi 2015:25; Kolarevic 2003:25; Woodbury 2010:11). Rather than drawing a house from the top down, so to speak, by adding roof, walls, doors, and windows, parametric design software lets architects describe formulas for the relationships between walls, the size of doors, and the distance in-between windows and then explore the possibilities offered by these relationships. As the size and complexity of the design grows, so does the relationships declared in its digital model, and the model becomes a new environment for keeping track of and exploring the interdependence of the many factors shaping the design of a given building. In the architecture studio, this associative logic becomes key to digital design. The explicit work with geometry as data means that digitally minded architects not only have new powers to create and control complex geometry but also new data-driven interfaces for collaborating internally and externally. 
This mediation of architectural imagination into new worlds through mathematics, digital computation, computer graphics, and software provides a case for digital materiality. Digital materiality goes beyond the mediating powers of technology disclosing new world to architects as posited by material hermeneutics by arguing that digital models quite literally form part of creative mind for architects. Creative agency arises as an emergent property dependent on the dynamic couplings between architects, software, their configuration and underlying digital materiality, studio organisation, project-specific collaborative relationships and so on (Poulsgaard and Clausen 2018; Poulsgaard 2020). In our relational perspective on agency, the specifics of these technological relationships form part of the ecologies of digital design from which architects' agency and imagination emerge. Not all mathematical constructs for manipulating computer graphics are equally useful for different tasks. Some software lends themselves easier to specific design operations when compared with others. Shifting user interfaces and the move from 2 to 3D in computer graphics change the work of architects. The point here is not to argue for the relative merits of this or that mathematical construct, software, or interface but rather that they matter and that the way they are coupled together forms a digital materiality of opportunities and resistances that fundamentally shape mind in digital design. They become integral to the architects' extension of creative agency into new imaginative worlds.

\subsection{Digital materiality in contemporary architectural design}

We argue that design and imagination in architecture are critically reliant on digital technology and different materials registers ranging from the molecular to the digital.

On a molecular level computation relies on materials such as gold, copper, aluminium, magnesium, silicon, zinc, and plastics (Blanchette 2011). These precious metals and plastic alloys are vital for the networks of wires, servers, and optical drives making digital parametric design possible. The materiality of computers has only had about 7 decades since the ENIAC computed its first equations to make their presence felt yet have done so with accelerating force. Exponentially growing, their powers reach beyond the molecular to also include the informational. And as we have seen, the informational in computation is mediated by successive layers of mathematics, software, and interfaces. We analyse this mediation through the lens of digital materiality.

Digital materiality emerges in the interplay between architects, molecular materials, programming languages, their algorithms, software, scripts, and mathematics. Just as molecular materials act as resistance and come to transform action upon material objects-as in the case of the potter at the wheel-so digital materiality comes to enable and transform our actions upon computers. As the close relationship between the development of mathematical splines in computer graphics, their underlying polynomial equations, and curvilinearity in architecture shows, digital materiality is fundamentally shaping creative imagination in architecture.

The notion of digital materiality is not new in architecture theory, but earlier conceptions differ in goals and scope from what we seek to develop.

Architecture historian Antoine Picon (2010) discusses materiality in digital design as a question of technically and culturally mediated perception, advancing a relational perspective on culture and technology that shares much with Ihde's PP. Interestingly, Picon likens digital design to clay modelling (ibid: 150), but he remains mainly interested in the larger cultural shifts introduced by computers in architecture and less in the relationships between specific software and design practices.

Architects Fabio Gramazio and Matthias Kohler, who lead the influential Robotic Fabrication Laboratory at ETH Zurich, define digital materiality explicitly in this way:

'We use the term digital materiality to describe an emergent transformation in the expression of architecture. Materiality is increasingly being enriched with digital characteristics, which substantially affect architecture's physis. Digital materiality evolves though the interplay between digital and material processes in design and construction.' (Gramazio and Kohler 2008:7, emphasis in original).

Gramazio and Kohler use the term to describe the integration of fabrication constraints and programming in the design process. This marks a shift in focus, they write, from the design of objects to the computational design of processes that will bring these objects into being in the physical world. Gramazio and Kohler (2008) write that digital materiality bridges the worlds of material properties and digital logic, but do not expand on how the digital comes to influence creative mind in design.

Architects Achim Menges and Sean Ahlquist have advanced the concept of computational design thinking to describe design practice oriented towards constructing computational systems leveraging clearly defined parameters and associative logics to explore the limits of technics and form (Menges and Ahlquist 2011). They propose that a deep understanding of how these systems operate, as form (e.g. on-screen drawings and geometry) and as mathematical ordering constructs (e.g. algorithmic functions), is fundamental to computational design thinking. In these practices, the position of the designer is changing explains Menges and Ahlquist, as computational design relies on the purposeful creation and execution of rules for the development of form, but not exhaustive descriptions of final forms themselves.

Gramazio and Kohler (2008) and Menges and Ahlquist (2011) are pioneers in digital design and fabrication. 
Alongside Picon's (2010) larger cultural history, their work highlights how a technologically mediated shift from finished object to processes, from free-form design to rulebased exploration, is at the heart of much design today. These perspectives present a challenge for understanding exactly how imagination and creativity emerge in computational design. We seek to meet this challenge through our own concept of digital materiality but do so through an expansion in focus, from describing how molecular materials or mathematical logics become the object of computational design to also discussing how the affordances of specific mathematical constructs, software, and interfaces come to influence the designer.

\section{Discussion: complexity, discovery, and evaluation in digital design}

Picon (2010), Gramazio and Kohler (2008), and Menges and Ahlquist (2011) argue that architects' creative imagination is bound up in interaction with systems reaching across material and informational realms. Along with them, we argue that if we want to understand architects designing with digital modelling environments, we must understand how cultural as well as mathematical, computational, and technical configurations of these environments come to shape their use. And we propose the concept of digital materiality as a framework for this analysis.

Digital materiality champions a relational approach to the analysis of technical practice. In this perspective, human creative thinking becomes a fundamentally situated activity where mind emerges through dynamic interactions between materials, technology, and culture and can never be the property of any of these elements alone. Advancing our understanding of computational design thinking via digital materiality suggests that architects' imagination in digital design does not arise from manipulation of mental plans and representations based in abstract computational logics, but rather emerges from within the interplay of forces in a given design situation mediated by computation and software.

The creation of form through digital design requires an understanding of how computational systems 'as form and as mathematical ordering constructs' operate (Menges and Ahlquist 2011), and how this 'bridges the worlds of material properties and digital logics' (Gramazio and Kohler 2008). But if we follow the relational ontology of MET and PP, which is also inherent in Picon (2010), this understanding must be embodied, practical and historical; it is made possible by the establishment of specific socio-technical and cultural environments that support creative computational design thinking through rich variation in interactions between parts. When analysing processes of digital design, we can neither reduce our explanations to the creative genius of the individual designer nor to the cold hard logic of computational tools; rather we should see how design intent and experience flow together in situated material engagement with specific computational design environments. Digital materiality seeks to capture this domain of possibilities while advancing a framework for analysing the entwining of creative agency, mind, and digital technics.

\subsection{Creative material engagement-getting a handle on complexity}

An implicit claim from MET, is that we use material and conceptual tools to recast abstract problems into more meaningful and manipulable terms (see also Kirsh 2010). At the heart of digital design, explains Gramazio and Kohler (2008), is the purposeful integration of difficult material and informational problems. These vary from project to project but can include: the description, control, and limits of advanced geometry (Burry 2011); control code for robots or 3D printers bringing digital form into the physical world (Søndergaard et al. 2016, Brander et al. 2016, Grigoriadis 2016); the physical behaviour of building structure, tectonics, and materials (Menges 2012; 2015); simulation of relationships between architectural form, daylight, and human well-being (Amundadottir et al. 2017). The list goes on as computational modelling and scripting lend themselves to near-endless development and variation (see Kolarevic 2003; Terzidis 2006). At heart, they work by providing a computational environment for integrating heterogeneous concerns into efficient digital models that structure architectural project development and collaboration between partners (Poulsgaard and Clausen 2018; Clausen et al. 2020).

By integrating geometry and computation in a visual interface, digital design models help architects and their collaborators mix vast amounts of data and recast problems from abstract mathematics and complex 3D geometry into something more manageable. Something giving designers a handle on the constraints presented by whatever heterogeneous forces their projects reckon with while allowing them to intuitively explore impacts of these along aesthetic lines. We will argue that we can understand this as a paradigm case of digital materiality.

Parametric design gave architects new tools for explicitly defining relationships between e.g. form, structure, and performance in their projects. This associative logic is at the heart of parametric design software and allows designers to integrate and manage complexity and growing amounts of data. In turn, this integration produces more data that can be used as input for new operations and so on. Crucially, different software and scripting interfaces allow for a multiplicity of visual representations and ways of manipulating the model. The ability of the digital architect to bring heterogeneous relationships together in a working whole, run 
calculations, transform geometry to data and back again, and manage vast arrays of parameters and their mathematical links thereby rides on the affordances of specific software and their underlying mathematical configuration. ${ }^{2}$

We argue that the efficacy of digital architectural models lies in their capacity to permit research into dynamic and complex systems at a humanly meaningful scale (Poulsgaard and Malafouris 2017). From this perspective, the success of digital modelling environments lies in the way they allow architects to selectively zoom in on, mix and process some aspects of the model while completely ignoring others (see also Cross 2006:37; Kallinikos 2005:189). Due to the growing power of computation from the 1990s onward and the near-instantaneous feedback between input and visual output in even complex models today, they further lend themselves to explorative discovery not dissimilar to the creative process of pot-making put forward by Malafouris to explain material agency and mind.

\subsection{Variation and discovery-extending possibilities for creative imagination}

Both Gramazio and Kohler (2008) and Menges and Ahlquist (2011) describe the main concern of digital design as the design of computational environments that enable the creation of form rather than the explicit design of form itself. Parametric design models have allowed architects to grow the complexity of parameters and relationships they work with in these environments to dizzying effect.

And implicit claim from PP is that our technically mediated being in the world is infinitely richer and more varied than a purely instrumental logic can account for. The world is a hot mess of impressions competing for our directed attention, demanding certain fitting attitudes to be adopted (Merleau-Ponty 2012:222), and the world qua technology is no different (Ihde 2009).

In the complex models of digital design, this messy tangle of relationships and potentiality lends itself to ongoing variation and creative discovery. The behaviour of linked elements in large parametric models can be difficult to foresee as changes to dimensions at one end of the linked model can quickly cascade through its entirety with unpredictable consequences for the whole (Poulsgaard and Clausen 2018). While explicit declaration and encoding of material and mathematical rules is necessary to create these environments, the fast computation of these relationships and

\footnotetext{
${ }^{2}$ Recall the discussion above on the relationship between mathematical splines, graphical user interfaces, and computation in the rise of digital design in the 1990s. See also Poulsgaard (2020) for a detailed discussion of how the underlying mathematical constructs used by different design software to describe and manipulate on-screen geometry come to vitally influences creative possibilities for designers.
}

the advent of intuitive graphical user interfaces enable an explorative approach to design to sit alongside the seemingly more logical computational one.

Once a digital model or software environment is created, different relationships can be generated by the drag of a mouse or the scripting of a few lines and their results for the whole can be looked at in beautifully rendered 3D. The model can be turned around, zoomed in on at one second to inspect a specific detail, and out of at the next to look at the whole. This potential for variation and fluid evaluation across scales is a result of digital materiality, the way successive layers of mathematics, software and interfaces enable and constrain embodied design interactions. In this, digital models become a means for creative discovery and imagination as much as for problem-solving in a strictly computational sense (see also Menges and Ahlquist 2011:10-11).

How do architects make sense of this vast possibility for variation borne from digital materiality? Picon (2010) and Carpo (2011) are explicit in their focus on the intricate relationship between technology and culture, but Gramazio and Kohler (2008:10) also situate their discussion of digital design within a long term context of cultural evolution writing that: "The digital is an independent cultural achievement resulting from centuries of human engagement with logic." Menges and Ahlquist (2011:11) write: "To fully clarify the definition of computation, it must be placed within the context of architectural practice, theory and technology." This interdependence between technology, practice, and culture is key for understanding digital design as both a purposeful and creative practice.

The impetuous of PP is that the deeply subjective focus of earlier phenomenology obscures the larger social and cultural reality of human experience. Ihde suggests that we cannot view technical practice in isolation but should see it in relation to specific embodied practices reproducing organisational, cultural, and social mores. He uses the word 'technoculture' to describe these relationships, arguing that technology embodies essential aspects of the culture of their production and use (Ihde 1993:57). As mentioned, Carpo (2011:40) suggested as much with the quip that few architects would have engaged deeply with calculous without the influential work of Gilles Deleuze. The point is that the embodied sense-making required by architects to evaluate the results of computational variation in their models is socially and culturally bound as well as technically and materially mediated. The concept of digital materiality radicalises the approach of material hermeneutics by locating creative agency not within the individual person, but in the embodied couplings of person, software, and its interfaces, configurations, code, and mathematics. In this sense, it brings the field of enactive mind into PP's study of twenty-first century technoscience 
(cf. Ihde and Malafouris 2019). Digital materiality further seeks to expand the reach and scope of MET to also encompass digital technologies and environments which are becoming ubiquitous to most aspects of life and work, thereby opening a new field of study for mind and material engagement (cf. Aydin et al. 2019).

\section{Conclusion}

The emergence of digital architectural design raises a number of questions for the hermeneutics of technology as digital tools not only introduce new computational possibilities for creation of form but also change the practices of design. Questions raised by digital design centre on the role of software in contemporary architectural practice, the relationship between architects and their digital tools, and above all on the way computational models act as mediums for creativity in design. Variants of these questions fuel dynamic discussions within the field of architecture; digital design theory is informed by constantly evolving practice and is in vigorous flux, as we have seen (Picon 2010; Carpo 2011; Gramazio and Kohler 2008; Menges and Ahlquist 2011). A basic assumption for the discussions cited here, is that designers are facing a new technologically mediated terrain which thoroughly changes design thinking (see also Oxman 2008; Rowe 2017). Here, we argue that answers to the questions raised by these discussions reach well beyond the fields of architecture and design as they require a wider definition of our relationship with emerging digital technologies.

PP (Ihde 1990, 1993, 2009, 2012; Ihde and Malafouris 2019) maintains that human beings and the world co-constitute each other and argues that we should pay particular attention to how technologies mediate these co-constitutive relationships. Following this, we have approached the relation between software and architect with what anthropologist Don Ihde (1993:3) has called an "instrumental realism," taking the correlate between technology and people as the starting point for understanding creativity in digital architectural design. This approach has been further developed via the framework of MET (Malafouris 2013a, 2014, 2015, 2019; Poulsgaard and Malafouris 2017; Poulsgaard 2019, 2020; Walls and Malafouris 2016), which sees creativity and mind as situated and enactive, dependent on material engagement and shifting interactional dynamics.

PP and MET position themselves against dualisms such as mind and body, individual and community, action and context and forward a holism that sees mind as embedded and extended in the relations between people and their environments. The relational ontology developed through these frameworks finds its analytical corollary in the concept of digital materiality.

We define digital materiality (Poulsgaard 2020) as the way successive layers of mathematics, software, and interfaces come to mediate and shape architects screen-based creative work. Malafouris (2014) has used the example of the potter at the wheel to explain how creative mind is inextricable from embodied engagement with technologies and materials. The potter at the wheel finds form through continuous interactions between body, wheel, and clay. Here, we argue that processes of creativity and mind are similar for the analogue potter at his wheel and the digital architect in front of her screen. The digital architect explores the materiality and possibilities inherent in her computational 3D models through embodied interaction with their interfaces and software. As in the example of the potter using the wheel to probe the materiality of his clay, the digital architect finds form through skilled engagement with the digital modelling environment across its interfaces, software, and their underlying mathematics. Interdependencies between these elements are brought forth through embodied practice and come to shape creative mind in digital design in much the same way as the materiality of the clay and wheel come to shape creative mind for the potter. Adding a digital dimension to the enactive analysis of MET, the concept of digital materiality helps explain how creative mind emerges in computational design thinking through rich embodied engagement within shifting physical and digital material registers.

PP shows the importance of situating these material engagements within their larger historical, social, and cultural contexts. PP also teaches us that there are ambiguities inherent in any mediated relationship between perceiver and world. In digital design, these ambiguities are made manageable, but also more complex, through the growing powers of computation. The complexity of contemporary digital design models forms a space for ongoing enactive discovery and creative material engagement for architects through manipulation of elements in the models and their underlying mathematical relationships. By shifting relationships within the digital model, architects can direct their attention towards widely different aspects of the design in question. In this perspective, digital design becomes a fundamentally situated activity where mind emerges through dynamic interaction between a variety of embodied, material, and cultural domains. Technology is both tekhne and logos, skill and learning, and both are socially mediated and subject to the interests and concerns of specific groups at specific points in time.

Digital materiality takes this seriously by arguing that creativity in digital design practice is an embodied activity where mind emerges from dynamic interactions with materials, technics, and culture and can never be the property 
of any of these elements alone. As recognised by architecture historians and practitioners such as Picon (2010), Carpo (2011), Gramazio and Kohler (2008), and Menges and Ahlquist (2011), digital design requires some understanding of all these realms. Embodied material engagement is where they come together to create meaningful connections through the ongoing sense-making between designer, model, software, and the wider field of architecture as both historical discipline and contemporary practice. Through these couplings, anchored and made possible by digital materiality, the embodied practice and cultural experience of the designer come to shape creative and social extension of mind in digital design.

Acknowledgements The writing of this article was supported by the Economic and Social Research Council (award number ES/J500112/1), and Clarendon Foundation, and St. John's College Oxford scholarships awarded to K. Poulsgaard. The writing of this article was supported by the European Research Council Consolidator Grant HANDMADE (No. 771997; European Union Horizon 2020 programme) awarded to L. Malafouris.

Open Access This article is licensed under a Creative Commons Attribution 4.0 International License, which permits use, sharing, adaptation, distribution and reproduction in any medium or format, as long as you give appropriate credit to the original author(s) and the source, provide a link to the Creative Commons licence, and indicate if changes were made. The images or other third party material in this article are included in the article's Creative Commons licence, unless indicated otherwise in a credit line to the material. If material is not included in the article's Creative Commons licence and your intended use is not permitted by statutory regulation or exceeds the permitted use, you will need to obtain permission directly from the copyright holder. To view a copy of this licence, visit http://creativecommons.org/licenses/by/4.0/.

\section{References}

Amundadottir M, Rockcastle S, Mandana SK, Andersen M (2017) A human-centric approach to assess daylight in buildings for nonvisual health potential, visual interest and gaze behavior. Build Environ 113:5-21

Aydin C, Woge MG, Verbeek PP (2019) Technological environmentality: conceptualizing technology as a mediating milieu. Philos Technol 32:321-338. https://doi.org/10.1007/s13347-018-0309-3

Blanchette J-F (2011) “A Material History of Bits.” J Am Soc Inform Sci Technol

Brander D et al (2016) Designing for hot-blade cutting: geometric approaches for high-speed manufacturing of doubly-curved architectural surfaces. In: Adriaenssens $\mathrm{S}$ et al (eds) Advances in architectural geometry. Zurich, pp 306-327

Brooks R (1991) Intelligence without reason. Artif Intell 47:139-159

Burry M (2011) Scripting cultures: architectural design and programming. Wiley, Chichester

Carpo M (2011) The Alphabet and the Algorithm. MIT Press, Cambridge

Carpo M (2012) The digital turn in architecture 1992-2012. Wiley, Chichester

Chemero A (2009) Radical embodied cognitive science. MIT Press, Cambridge
Clark A, Chalmers D (1998) The extended mind. Analysis 58(1):7-19

Clausen K, Lineham S, Lund M, Rahbek L, Poulsgaard KS (2020) "New Workflows and Collaborations." In: Sheil B, Thomsen M, Tamke M, Hanna S (eds) Design transactions. Rethinking information modelling for a new material age. UCL Press, London

Clowes RW (2018) Immaterial engagement: Human agency and the cognitive ecology of the internet. Phenomenol Cogn Sci. https:// doi.org/10.1007/s11097-018-9560-4

Clowes RW (2019) Screen reading and the creation of new cognitive ecologies. AI Soc 34(4):705-720. https://doi.org/10.1007/s0014 6-017-0785-5

Cross N (2006) Designerly Ways of Knowing. Springer, London

Davis D (2013a) A history of parametric. Available at: https://www. danieldavis.com/a-history-of-parametric/ Accessed 15.12.2019

Davis D (2013b) Modelled on software engineering: flexible parametric models in the practice of architecture. PhD Dissertation. Melbourne: School of Architecture and Design, RMIT University

Deleuze G (1988) The Fold: Leibniz and the Baroque. Athlone Press, London

Di Paolo E (2009) Extended Life. Topoi 28:9-21. https://doi. org/10.1007/s11245-008-9042-3

Dreyfus HL (2002) Intelligence without representation-MerleauPonty's critique of mental representation The relevance of phenomenology to scientific explanation. Phenomenol Cogn Sci 1(4):367-383. https://doi.org/10.1023/A:1021351606209

Dyson G (2012) Turing's Cathederal: The Origins of the Digital Universe. Pantheon Books, New York

Farin GE (2002) A history of curves and surfaces in CADG. In: Farin GE, Hoschek J, Kim M Handbook of Computer Aided Geometric Design. Elsevier, Amsterdam, pp. 1-22

Gallagher S (2017) Enactivist interventions, rethinking the mind. Oxford University Press, Oxford

Gallagher S, Allen M (2018) Active inference, enactivism and the hermeneutics of social cognition. Synthese 195(6):2627-2648. https://doi.org/10.1007/s11229-016-1269-8

Gell A (1998) Art and Agency: An Anthropological Theory. Clarendon, Oxford

Gramazio F, Kohler M (2008) Digital Materiality in Architecture. Lars Müller Publishers, Zurich

Grigoriadis K (2016) Mixed Matters. A multi-material design compendium. Jovis, Berlin

Hutchins E (2010) Cognitive ecology. Topics Cogn Sci 2(4):705-715

Hutto D, Myin E (2013) Radicalizing Enactivism. Basic minds without content. MIT Press, Cambridge

Ihde D (1990) Technology and Lifeworld: From garden to earth. Indiana University Press, Bloomington

Ihde D (1993) Postphenomenology. Essays in the Postmodern Context. Northwestern University Press, Evanston

Ihde D (1999) Expanding Hermeneutics: Visualism in Science. Northwestern University Press, Evanston

Ihde D (2009) Postphenomenology and Technoscience: The Peking University Lectures. SUNY Press, Albany

Ihde D (2012) 'Cartesianism' Redux or Situated Knowledges. Found Sci 17(4):369-372. https://doi.org/10.1007/s10699-011-9243-x

Ihde D, Malafouris L (2019) Homo faber revisited: postphenomenology and material engagement theory. Philos Technol 32(2):195214. https://doi.org/10.1007/s13347-018-0321-7

Kallinikos J (2005) The order of technology: complexity and control in a connected world. Inf Organ 15:185-202

Kolarevic B (2003) Architecture in the Digital Age: Design and Manufacturing. Spon, New York

Koukouti MD, Malafouris L (2020) Material imagination: An anthropological perspective. In: Abraham A (ed) The Cambridge handbook of the imagination. Cambridge University Press, Cambridge, pp 30-46 
Kirsh D (2010) Thinking with external representations. AI Soc 25:441454. https://doi.org/10.1007/s00146-010-0272-8

Lynn G (1993) "Folding in Architecture." In Carpo (ed.) (2012) The Digital Turn in Architecture 1992-2012. John Wiley and Sons

Malafouris L (2008) At the Potter's Wheel: an argument for material agency. In: Knappett C, Malafouris L (eds) Material agency. Springer, Boston, MA. https://doi.org/10.1007/978-0-38774711-8_2

Malafouris L (2009) Vital Materiality/Biothing. In: Andrasek A (ed) Biothing. Editions HYX, Collection FRAC centre, pp 32-47

Malafouris L (2011) Enactive discovery: The aesthetic of material engagement. In: Manzotti R (ed) Situated aesthetics: Art beyond the skin. Imprint Academic, Exeter, pp 123-141

Malafouris L (2013a) How Things Shape the Mind: A Theory of Material Engagement. MIT, Cambridge

Malafouris L (2013) On thinking and form-making. In: Brayer MA, Migayrou F (eds) Naturaliser Architecture/Naturalizing Architecture. Editions HYX, Collection FRAC centre, pp 244-253

Malafouris L (2014) Creative thinging: The feeling of and for clay. Pragm Cogn 22(1):140-158

Malafouris L (2015) Metaplasticity and the primacy of material engagement. Time Mind 8(4):351-371

Malafouris L (2016) Hylonoetics: On the priority of material engagement. In: Grigoriadis K (ed) Mixed Matters: A Multi-Material Design Compendium. Jovis Verlag, Berlin, pp 140-146

Malafouris L (2019) Mind and material engagement. Phenomen Cogn Sci 18(1):1-17. https://doi.org/10.1007/s11097-018-9606-7

Malafouris L (2020) Thinking as "Thinging": Psychology With Things. Curr Direc Psychol Sci 29(1):3-8

Menges A (2015) Special Issue: Material Synthesis: Fusing the Physical and the Computational. Architectural Design 85(5):1-136

Menges A (2012) Material Computation: Higher Integration in Morphogenetic Design. John Wiley \& Sons, Chichester

Menges A, Ahlquist S (2011) Computational Design Thinking. John Wiley \& Sons, Chichester

Merleau-Ponty M (2012) [1962] Phenemenology of Perception, 2nd edn. Routledge, London

Newell A, Shaw JC, Simon H (1959) "The process of creative thinking." Presented at a Symposium on Creative Thinking, University of Colorado, Boulder, Colorado, May 16, 1958

Oxman R (2008) Digital architecture as a challenge for design pedagogy: theory, knowledge, models and medium. Des Stud 29:99-120

Oxman R, Oxman R (2014) Theories of the Digital in Architecture. Routledge, London

Pallasmaa J (2017) Embodied and existential wisdom in architecture: the thinking hand. Body Soc 23(1):96-111

Penny S (2017) Making sense: cognition, computing, art, and embodiment. MIT Press, Cambridge

Picon A (2010) Digital Culture in Architecture: An Introduction for the Design Professions. Birkhäuser, Basel

Poulsgaard KS (2019) Enactive individuation: technics, temporality and affect in digital design and fabrication. Phenom Cogn Sci 18:281-298. https://doi.org/10.1007/s11097-017-9539-6
Poulsgaard KS, Malafouris L (2017) Models, mathematics and materials in digital architecture. In Cognition beyond the brain (pp. 283-304). Springer International Publishing, Cham

Poulsgaard KS, Clausen K (2018) Modelling workflow: Data, Collaboration, and Dynamic Modelling Practice. In De Rycke et al. Humanizing Digital Reality: Design Modelling Symposium Paris

Poulsgaard KS (2020) Digital Materiality: creativity and social mind in digital modelling at 3XN Architects and GXN Innovation. PhD dissertation School of Anthropology and Museum Ethnography, University of Oxford, Oxford

Rietveld E, Brouwers AA (2017) Optimal grip on affordances in architectural design practices: An ethnography. Phenomenol Cogn Sci 16:545-564. https://doi.org/10.1007/s11097-016-9475-x

Rowe PG (2017) Design Thinking in the Digital Age. Sternberg Press, Harvard

Sigaut (1994) "Technology." Companion Encyclopaedia of Anthropology: Humanity, Culture and Social Life. Ed. Tim Ingold. Routledge

Simon HA, Newell A (1971) "Human Problem Solving: The State of Theory in 1970." Am Psychol. 145-159

Stiegler B (1998) Technics and Time, 1: The Fault of Epimetheus. Stanford U, Stanford

Sutherland IE (2003) [1963] Sketchpad: A Man-Machine Graphical Communication System. Technical Report number 574, University of Cambridge Computer Laboratory

Søndergaard A et al (2016) "Robotic Hot-Blade Cutting." In Robotic Fabrication in Architecture, Art and Design. (ed). Rheinhardt et al. Springer: 151-166

Tedeshi A (2015) AAD_Algorithms-Aided Design: Parametric Strategies Using Grasshopper. Edizioni Le Penseur, Napoli

Terzidis K (2006) Algorithmic Architecture. Architectural, Oxford

Thompson E (2007) Mind in Life. Biology, Phenomenology, and the Sciences of Mind. Harvard University Press, Cambridge

Tripathi AK (2017) Hermeneutics of Technological Culture. AI Soc 32:137-148. https://doi.org/10.1007/s00146-017-0717-4

Tripathi AK (forthcoming) Editorial to AI SOC special issue, MHTC van Dijk L, Rietveld E (2018) Situated anticipation. Synthese Adv Online Publ. https://doi.org/10.1007/s11229-018-02013-8

van Gelder T (1995) What might Cognition be if not Computation? J Philos 91:345-381

Varela FJ, Thompson E, Rosch E (1991) The Embodied Mind: Cognitive Science and Human Experience. MIT, Cambridge

Walls M, Malafouris L (2016) Creativity as a developmental ecology. In: Glaveanu VP (ed) The Palgrave handbook of creativity and culture research. Palgrave Macmillan, Basingstoke, pp 553-566

Woodburry RF (2010) Elements of Parametric Design. Routledge, London

Publisher's Note Springer Nature remains neutral with regard to jurisdictional claims in published maps and institutional affiliations. 